\title{
Mycophenolate Mofetil (Cellcept) Induced Pneumonitis: Case Report and Review of Literature
}

\author{
Iboro Udoete, MD.1 ${ }^{1}$, Ngozika Orjioke MD., FCCP2, \\ Okelue Edwards Okobi MD. M. Sc ${ }^{3}$ and Chinwendu Onuegbu, $\mathrm{MD}^{4}$
}

\author{
${ }^{1}$ Master of Public Health,Central Michigan University, Michigan, USA \\ ${ }^{2}$ Covenant Pulmonary Critical Care, East Point, Georgia, USA \\ ${ }^{3}$ Lakeside Medical Center, Belle Glade, USA \\ ${ }^{4}$ All Saints University, Dominica
}

*Corresponding author details: Iboro Udoete, MD.; udoet1io@cmich.edu

\section{LEARNING OBJECTIVES}

- Recognize the clinical features of medication-induced pneumonitis

- Assess for the use of anti-inflammatories masking early symptom presentation

\begin{abstract}
Mycophenolate is used to treat several disease conditions. Several side effects have been reported in several reported cases; however, a few other side effects have been underreported, for example, post-transplant pneumonitis. This case demonstrates suspected mycophenolate-associated pneumonitis, which was masked by the concurrent immune-modulatory drugs that the patients were taking. The pneumonitis became evident after the withdrawal of the immune drugs. This seldomly reported pneumonitis and mycophenolate association calls for further vigilance and a high index of suspicion by physicians when pneumonitis develops in patients taking this valuable medication.
\end{abstract}

Keywords: mycophenolate; pneumonitis; medication

\section{INTRODUCTION}

Mycophenolate Mofetil (Cellcept) has been reported to cause a vast number of adverse effects, including reactivation of latent $\mathrm{TB}$, increased susceptibility to recurrent infections, sepsis, increased risk of lymphoma, leukopenia, pulmonary hypertension, etc. (Hosohata et al., 2018, Behrend M, 2001). The adverse effect of Mycophenolate Mofetil (Cellcept), least spoken about, plausibly because of its rarity, is drug-induced pneumonitis. We report a case of pneumonitis associated with Mycophenolate use after kidney transplantation.

\section{CASE}

A 45-year-old African American female with a history of hypertension and end-stage renal disease presented to the clinic status-post-living- donor renal transplant for a followup visit. During this visit, she reported nasal congestion, shortness of breath on exertion, cough, and wheezing. She denied chest pain, palpitations, fevers, or chills.

The patient was started on Mycophenolate Mofetil (Cellcept) three weeks earlier post-surgically and denied having these symptoms prior to starting medical therapy. Her other medications included Bactrim and tapering doses of both Tacrolimus (Prograf) and Prednisone, which were also started post-surgically. Her baseline medications included Albuterol sulfate as needed for seasonal allergies and Lisinopril. Immunizations were up to date, including the pneumococcal and influenza vaccine received earlier in the year.
On physical examination, there were crackles, "pops, and squeaks" on lung auscultation with accompanied wheezing. Cardiologic, abdominal, and neurologic examinations were all unremarkable.

Pulmonary function tests, 2D Echo, and high-resolution CT scan of the chest without contrast were ordered. The chest CT revealed; restrictive lung pattern with moderately reduced DLCO, subtle bilateral ground-glass mosaic attenuation with an incidental sub-centimeter lung nodule in the right upper lobe, the ECHO showed; mild pericardial effusion and an ejection fraction of 55\%. After a review of patients presenting symptoms, clinical signs, and CT scan imaging features, a diagnosis of pneumonitis was made with mycophenolate highly suspected as a causative factor. The patient was started on Fluticasone-Salmeterol and Mycophenolate was discontinued. During the subsequent two-week clinic visit, she reported moderate relief of the pulmonary symptoms, which further supported the diagnosis. A six-month follow-up was recommended to monitor the sub-centimeter lung nodule for progression in size.

\section{DISCUSSION}

This patient was diagnosed with Mycophenolate mofetil (Cellcept) induced pneumonitis. The symptoms presenting three weeks after initiation of Mycophenolate and a tapering dose of prednisone indicate that the antiinflammatory effect of prednisone blunted the pulmonary 
effects of the medication. As the dose of prednisone was slowly tapered in the subsequent weeks, there was a paradoxical increase in the severity of this patient's pulmonary symptoms.

Cases of Mycophenolate induced pneumonitis have been previously reported, but extensive clinical trials and research accessing its incidence are currently unreported. (Pocivalnik et al., 2013, Gross et al., 1997). Pneumonitis is a broad term that refers to inflammation of lung tissue. Technically, pneumonia is caused by a series of pneumonitis caused by infections. Pneumonitis, however, is typically used to refer to the noninfectious etiology of lung inflammation. Difficulty breathing, commonly accompanied by a nonproductive cough, is a frequent symptom of pneumonitis. Other common symptoms are fatigue, cough, loss of appetite, and weight loss. Treatment focuses on avoiding irritants and reducing inflammatory triggers like discontinuation of offending medications and chemicals.

Development of various interstitial lung diseases in patients on Mycophenolate is another rarely reported side effect, with only a few isolated case reports available. (Abelleira et al., 2018, Reynolds et al., 2008). Medication-induced pneumonitis has been reported to be caused by various chemotherapeutic agents, antibiotics, immunosuppressive agents, and anti-arrhythmic medications, especially amiodarone.

Medication-induced pneumonitis is a diagnosis of exclusion made after other differential diagnoses have been screened for and excluded. Patients normally improve after medication cessation, reduction of offending medication dosage, or a trial of glucocorticoid therapy.

\section{CONCLUSION}

Mycophenolate induced pneumonitis symptoms may present late in some patients due to simultaneous use of anti-inflammatory agents like prednisone, that mask symptoms and delay clinical presentation. Patients taking Mycophenolate should be closely monitored for symptoms of pneumonitis after drug initiation. Once symptoms are identified, dosage should be reduced or stopped to avoid further deterioration of lung function.

\section{CONFLICTS OF INTEREST}

None declared

\section{AUTHOR CONTRIBUTIONS}

Iboro Udoete, MD: assisted with patient care postsurgically during follow-up clinic visits and wrote the manuscript. Ngozika Orjioke MD, FCCP: provided postsurgical pulmonary clearance for the patient, Okelue Edwards Okobi, MD, M.Sc: article review and summary, Chinwendu Angel Onuegbu, MD: article review, referencing, and summary.

\section{ETHICAL APPROVAL}

Consent was gained from the patient on whom this case is based.

\section{REFERENCES}

[1] Behrend M. Adverse Gastrointestinal Effects of Mycophenolate Mofetil. Drug Safety. 2001;24(9). doi:10.2165/00002018-200124090-00002

[2] Hosohata K, Matsuoka E, Inada A, et al. Differential profiles of adverse events associated with mycophenolate mofetil between adult and pediatric renal transplant patients. Journal of International Medical Research. 2018;46(11). doi:10.1177/0300060518786917

[3] Pocivalnik M, Kirsch AH, Hassler EM, Rosenkranz AR, Eller K. Fever and pneumonitis induced by entericcoated mycophenolate sodium in a patient after kidney transplantation. Transplant International. 2013;26(7). doi:10.1111/tri.12104

[4] Reynolds BC, Paton JY, Howatson AG, Ramage IJ. Reversible chronic pulmonary fibrosis associated with MMF in a pediatric patient: A case report. Pediatric Transplantation. 2008;12(2). doi:10.1111/j.1399-3046.2007. 00707.x

[5] Abelleira R, Elena Toubes M, Suárez-Antelo J, Valdés L. Reversible diffuse lung disease after mycophenolate mofetil withdrawal. Pulmonology. 2018;24(6):361-362.

doi: 10.1016/j.pulmoe.2018.10.003

[6] Gross DC, Sasaki TM, Buick MK, Light JA. ACUTE RESPIRATORY FAILURE AND PULMONARY FIBROSIS SECONDARY TO ADMINISTRATION OF MYCOPHENOLATE MOFETIL. Transplantation. 1997;64(11). doi:10.1097/00007890-19971215000020 